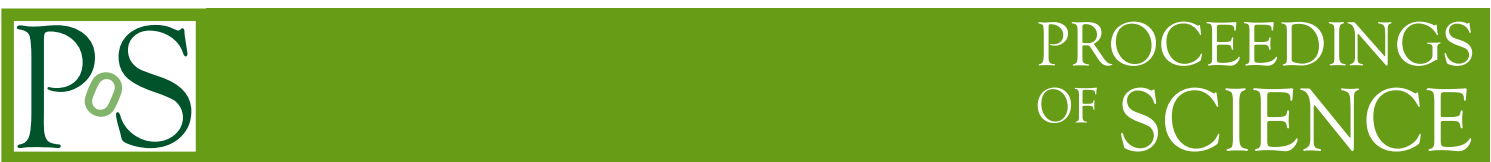

\title{
Charm (and bottom) baryons and charmonium excitations from the lattice*
}

\section{Padmanath ${ }^{a, *}$}

${ }^{a}$ Helmholtz Institut Mainz, Staudingerweg 18, 55128 Mainz, Germany GSI Helmholtzzentrum für Schwerionenforschung, Darmstadt (Germany)

E-mail: pmadanag@uni-mainz.de, papppan@gmail.com

This report discusses some recent investigations of the heavy hadron spectra using lattice QCD. The first half addresses multiple precision determinations of the masses of charm (and bottom) baryons. Recent lattice results in the tetraquark and the dibaryon sectors are also presented. The second half focuses on new exploratory studies of the excited charmonium spectra in the vector and scalar channels. Along the way, lattice results are compared with the experimental results, wherever they are available.

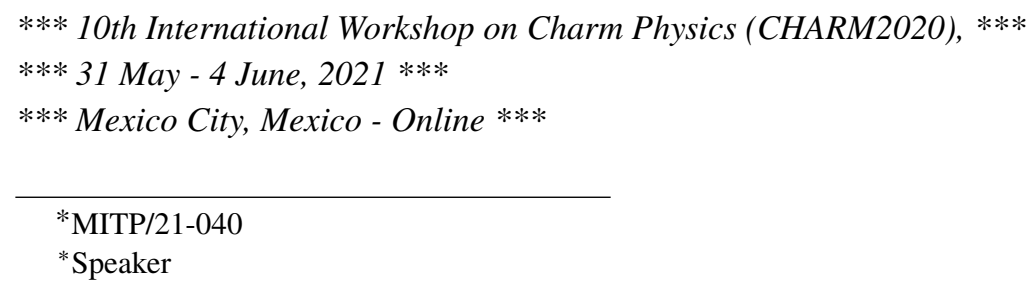




\section{Introduction}

The past decade has been splendid for heavy hadrons with several discoveries both in conventional and exotic channels. Among these are the doubly charmed baryon [1], the charmoniumnucleon pentaquark resonances [2], five narrow $\Omega_{c}$ resonances [3], and a handful of heavy hadrons with four quark content. Detailed accounts of various discoveries and the hadron properties can be found in recent reviews of this subject $[4,5]$. The expanding family of discovered heavy hadrons motivates theoretical investigations of such and similar excitations and their properties, which can be tested in experiments.

Remarkable progress in lattice QCD, which is an ab-initio non-perturbative approach that can be systematically improved to achieve complete control over all uncertainties, has allowed modern investigations to make precision measurements in QCD that are of phenomenological interest. This advancement is reflected in several lattice publications on different aspects of QCD, including the hadron spectrum determination in recent years (see Lattice yearly conference proceedings e.g. Ref. [6]). Most of the investigations are made in the isospin symmetric limit, where masses of up and down quarks are chosen to be the same, and with a heavier than physical light quark mass. More recently, studies are also performed directly at the physical values for several heavy flavor physics observables as presented in the FLAG review[7]. While many calculations utilize lattice QCD gauge configurations that account for the dynamics of light and strange quarks during the simulation $\left(N_{f}=2+1\right)$, some newer studies use ensembles that also incorporate the charm quark dynamics during the gauge field generation $\left(N_{f}=2+1+1\right)$. Significant efforts have also been put to include the strong and QED isospin breaking effects using lattice techniques, but for heavy hadrons such studies are limited [8].

Hadron spectroscopy using lattice techniques commonly proceeds through the extraction of finite-volume energy spectrum $\left(E_{n}\right)$ on a discretized space-time. This is achieved by computing two-point correlation functions

$$
C_{i j}\left(t_{f}-t^{\prime}\right)=\left\langle O_{i}\left(t_{f}\right) O_{j}^{\dagger}\left(t^{\prime}\right)\right\rangle=\sum_{n} \frac{Z_{i}^{n} Z_{j}^{n *}}{2 E_{n}} e^{-E_{n}\left(t_{f}-t^{\prime}\right)},
$$

where $O_{i}(t)$ is the hadronic current with the desired quantum numbers and $Z_{i}^{n}=\left\langle O_{i} \mid n\right\rangle$ is the operator state overlap. $O_{i}(t)$ couples to all the states including single-particle levels, and their radial excitations, as well as multi-particle levels with these quantum numbers. A standard practice to extract the energy spectrum is to compute matrices of correlation functions between a basis of interpolators with the same quantum numbers $[9,10]$ and to solve the Generalized EigenValue Problem (GEVP) [11]

$$
C_{i j}(t) v_{j}^{n}\left(t-t_{0}\right)=\lambda^{n}\left(t-t_{0}\right) C_{i j}\left(t_{0}\right) v_{j}^{n}\left(t-t_{0}\right) .
$$

$E_{n} \mathrm{~s}$ are extracted from the large $t$ behavior of the eigenvalue correlators $\lambda^{n}\left(t-t_{0}\right)$.

We classify the modern-day lattice calculations for hadron spectroscopy into two categories. In the first category, one computes the energy spectrum on the lattice and attributes the finite-volume energy levels to the strong interaction stable hadrons. The mass estimates are extracted directly from simple fits to the large $t$ behavior of the two-point correlation functions. A handful of ground-state baryons are stable with respect to strong decays, and their masses can be extracted precisely on the 
lattice. Multiple lattice groups have been performing detailed systematic investigations of stable hadrons. A summary and details of various such studies can be found in the reviews [12,13]. More complicated systems (such as hadrons close to the elastic threshold, tetraquark, and pentaquark systems that have received attention recently) are also treated on a similar footing as stable hadrons in several recent exploratory investigations on the lattice (see Section 3).

The trivial connection of the finite-volume energy levels with the infinite-volume spectrum is no longer valid for hadrons close to and above the elastic threshold, which falls into the second category. A standard procedure to extract the infinite-volume two-particle scattering amplitudes from finite-volume energy spectrum is through the quantization condition,

$$
\operatorname{det}\left(K^{-1}-B\right)=0
$$

first derived by Lüscher for elastic scattering of two spinless particles in the rest frame[14]. Here $K$ is related to the infinite-volume scattering amplitudes and $B$ is built out of known mathematical functions of the system's total energy $E_{c m}$. Given the finite-volume energy levels and a parametrization of $K$, one looks for the best-fit parameters that satisfy Eq. 3. The infinite-volume scattering amplitudes built from these best-fit parameters are then investigated across the complex s-plane for pole singularities related to discovered hadrons. Investigating the model independence of the pole positions in the extracted scattering amplitudes using different parametrizations of $K$ further demonstrates the robustness of the lattice determinations [15]. A detailed review on methodologies for treating the hadronic resonances on the lattice and various lattice calculations along these lines can be found in Ref. [16]. A summary of results from more recent calculations in the light hadron and heavy meson sector are discussed in the proceedings [17]. Recent theoretical and numerical advancements in treating three-particle scattering in the finite-volume can be found in Refs. [18-20].

This report presents results from some recent lattice determinations of the spectra of heavy hadrons. Section 2 focusses on heavy baryons, whereas section 3 on heavy four-quark and sixquark systems. In section 4 , we discuss a recent investigation of excited and exotic charmonium resonances and summarize in Section 5.

\section{Charm and bottom heavy baryons}

Precise determination of the energy epectra of hadrons which are stable under strong interactions are benchmarks for the lattice investigations. There exist several calculations that have predicted/postdicted masses of heavy hadrons with full control over the statistical as well as systematic uncertainties (For mesons, c.f. Ref. [21]). In Fig. 1, we present the recent lattice results for ground-state masses for singly and doubly charmed baryons (See Ref. [13] for references).

Singly charmed baryons : On the left side of Fig. 1, we present a summary of recent lattice results for the masses of singly charmed baryons. The gray horizontal lines are experimental masses. Different lattice investigations utilize different setups and procedures, and hence have different systematics. In spite of this, an excellent overall agreement between all the lattice estimates and the respective experimental values is evident for all the hadrons. Note that in the physical world, $\Sigma_{c}$, $\Sigma_{c}^{*}$, and $\Xi_{c}^{*}$ can decay via strong interactions. Even so, the lattice estimates without any rigorous amplitude analysis agree remarkably well with the experiment values for these channels. 

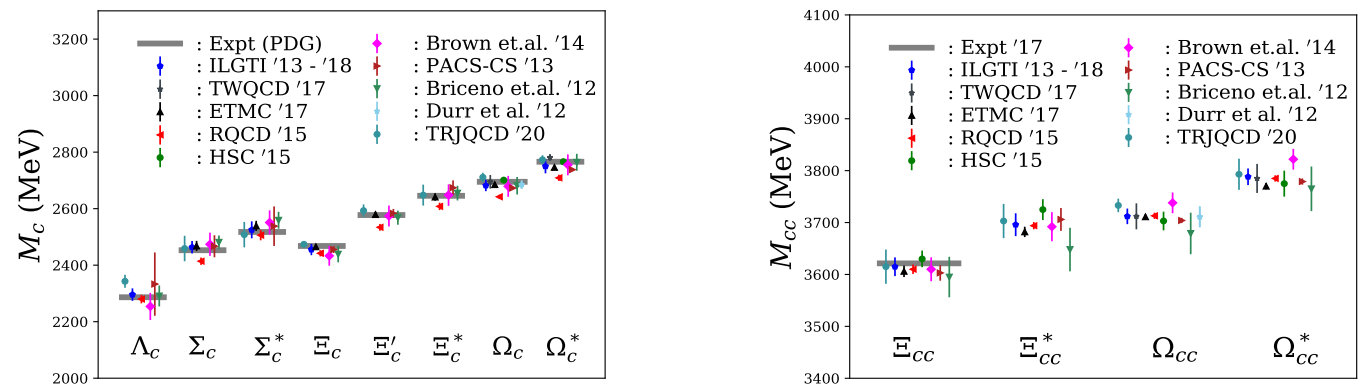

Figure 1: Left : A summary of various lattice results for single charmed baryon masses. The horizontal lines represent the experimental masses. Right : Lattice predictions for the masses of the doubly charm baryons. The experimental mass for $\Xi_{c c}\left(1 / 2^{+}\right)$as determined by LHCb [22] is shown as a horizontal band.
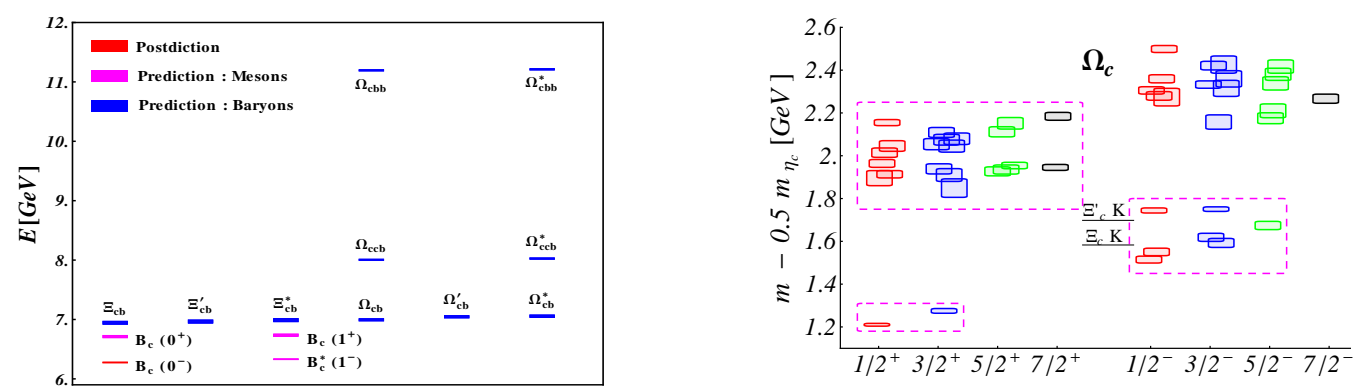

Figure 2: Lattice estimates for charmed-bottom hadron masses as determined in Ref. [24]. Excitation spectra of $\Omega_{c}$ baryon (right) as determined in Ref. [25].

Doubly charmed baryons : On the right side of Fig. 1, lattice predictions for the ground-state masses of doubly charmed baryons are presented. The only discovered doubly charmed baryon $\Xi_{c c}\left(1 / 2^{+}\right)[22]$ is presented by the horizontal line, which is consistent with all the lattice estimates. We emphasize that most of these lattice determinations were reported before the LHCb discovery and are all predictions. This demonstrates the potential of lattice QCD in making predictions in hadron spectroscopy calculations. The agreement between different lattice measurements indicates cut-off errors in these heavy hadron observables are small.

Another interesting point that we would like to mention is the comparison of lattice results with the SELEX candidate for a doubly charm baryon (3519(1) MeV) [23]. All lattice estimates for $\Xi_{c c}\left(1 / 2^{+}\right)$mass consistently lie $\sim 100 \mathrm{MeV}$ above the SELEX measurement. Furthermore, a lattice QCD+QED calculation with $N_{f}=1+1+1+1$ flavors performed by the BMW collaboration [8] indicates the mass difference between the isospin partners of $\Xi_{c c}\left(1 / 2^{+}\right)$to be $2.16(11)(17) \mathrm{MeV}$. Hence lattice investigations exclude any possibility of the SELEX measurement to be related to a doubly charmed baryon.

Charmed bottom baryons : On the left side of Fig. 2, we present lattice estimates for the ground-state masses of hadrons with at least one charm and one bottom quark studied on $N_{f}=2+1+1$ HISQ fermion MILC ensembles [24]. In this investigation, controlled chiral and continuum extrapolations are performed to obtain reliable predictions for many yet-to-bediscovered charmed-bottom hadrons. The mass estimate for $B_{c}(1 S)$ meson is consistent with the experimental value. The results for charmed bottom meson masses also agree with other 

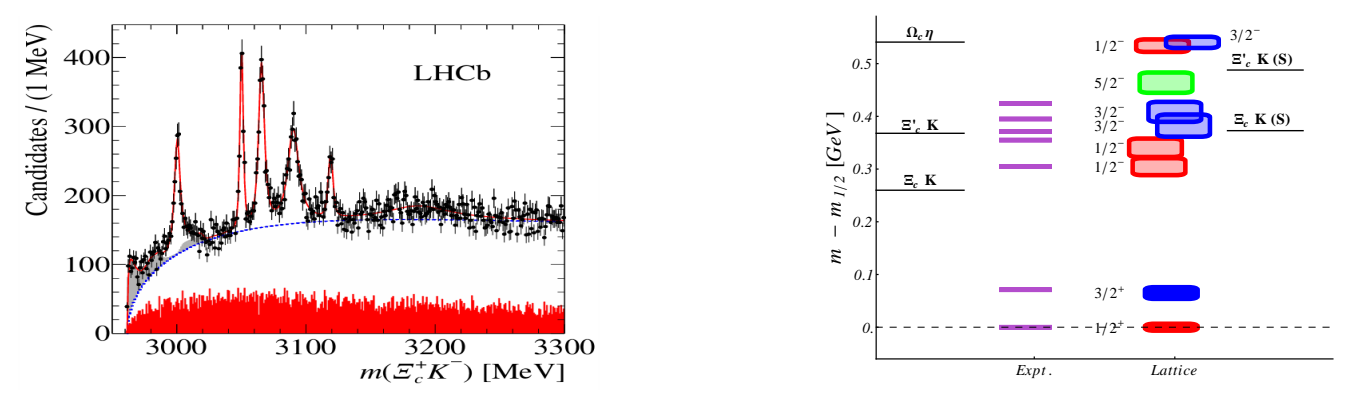

Figure 3: Left : (Figure adapted from Ref. [3]) The event distribution in the $\Xi_{c}^{+} K^{-}$final states with peaks inferred as excited $\Omega_{c}$ baryons by the LHCb Collaboration. Right : The finite-volume energy spectrum is compared with the experimental masses of $\Omega_{c}$ baryons. Relevant low lying scattering thresholds are shown on the left and the finite-volume non-interacting energy levels are shown on the right as black horizontal lines.

existing lattice determinations [26, 27]. The baryon mass estimates are also consistent with the only existing previous dynamical calculation [28] performed on ensembles with only two different lattice spacings.

Excited $\Omega_{c}$ baryons : Not only the ground-state energy determination but hadron spectroscopy using lattice techniques has also made remarkable progress in determining the excitation spectrum. To this end, a basis of carefully constructed interpolating operators are used to compute the correlation matrices (Eq 2 ) and variationally analyzed to extract the excitation energy spectrum. On the right side of Fig. 2, we present the excitation spectrum of $\Omega_{c}$ baryons in various $J^{P}$ quantum numbers [25]. Similar excitation spectra have also been determined using lattice QCD for other charmed baryons [29-31] and triply bottom baryons [32]. Given lattice results, the masses of recently discovered five excited $\Omega_{c}$ baryons by the LHCb Collaboration [3] can immediately be compared.

On the left side of Fig. 3, we show the discovery plot of excited $\Omega_{c}$ baryons by the LHCb Collaboration [3] displaying the related five narrow peaks. The only existing lattice estimates for the excited $\Omega_{c}$ baryons [25] are compared with the experimental values on the right side of Fig. 3. Working with a pion mass of $\sim 391 \mathrm{MeV}$ and relatively coarse lattice spacing, this calculation aimed at an exploratory determination of the excitation spectrum and hence does not address various systematics in depth. The $1 S$ hyperfine splitting in $\Omega_{c}$ baryons, which is known to be very sensitive to cut-off effects, can be seen to be consistent between the lattice estimate and the experimental value. Lattice QCD predicts five states in the energy range $(0.3,0.5) \mathrm{GeV}$, where the newly discovered $\Omega_{c}$ baryons lie. Qualitatively these five states correspond to the $p$-wave excitations of the $\Omega_{c}$ baryon. The authors of Ref. [25] also performed another lattice study with a different setup to check the extent of discretization uncertainties due to the coarse lattice spacing in the original calculation. Although the second study did not use a large interpolator basis to extract all the low lying excitations, it utilized three lattice QCD ensembles with different lattice spacings to make a controlled continuum extrapolation of the ground-states they extract. The second lattice study confirmed the pattern of states qualitatively. These lattice results were reported in several conferences such as Lattice 2014 [33] as well as in Charm 2013, 2015 [31, 34] and hence preceded the $\mathrm{LHCb}$ discovery. Recent $\mathrm{LHCb}$ report conflicts these quantum number assignments to these five 

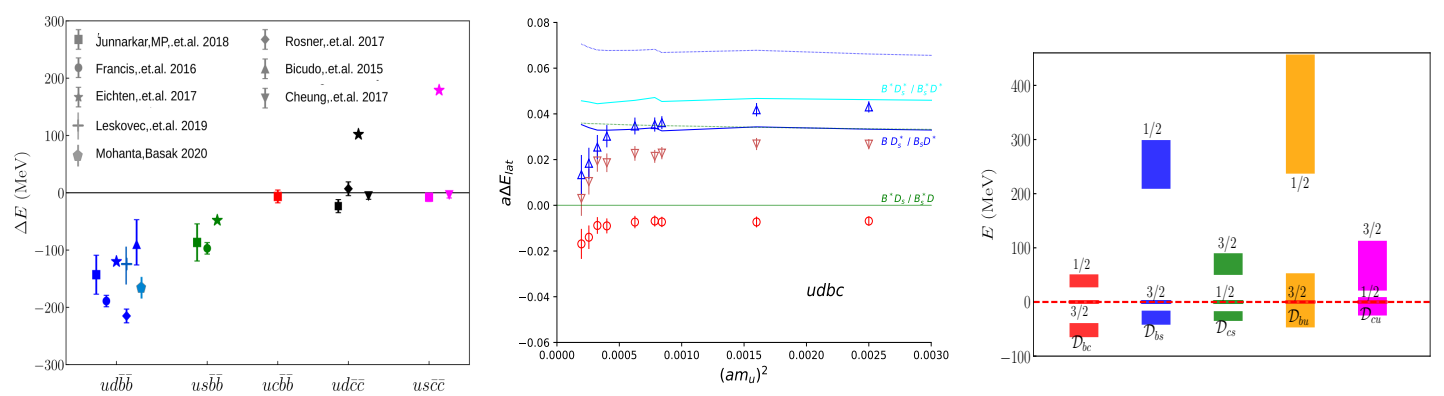

Figure 4: Left: Summary of all the lattice results and a selected set of non-lattice estimates for the groundstate energies in the doubly heavy tetraquark channels. Center: The light quark mass dependence of the finite-volume spectrum for the $\bar{b} \bar{c} d u$ four quark system by the ILGTI collaboration. Right: Low lying finite-volume spectra of Deuteron-like heavy dibaryons as determined in Ref. [38]

states [35]. This indicates the necessity of systematic lattice investigations involving the amplitude analysis on finite-volume spectrum using finer lattices to resolve the discrepancy quantitatively.

An obvious next step towards this is to include multi-hadron interpolators that are related to the nearby non-interacting baryon-meson levels and rigorous finite-volume analysis. Lattice calculations using baryon-meson interpolators are limited to a few. Most of them are in the light baryon sector (For more info see Ref. [13])). More recently, two studies investigated interactions in charmonium-nucleon systems [36, 37]. In Ref. [36], the authors studied the static $Q \bar{Q}$ potential in the presence of various light hadrons, including baryons. In all the cases, they found that bound state configurations are energetically favored. In Ref. [37], the authors reported the finite-volume spectra for nucleon- $J / \psi$ and nucleon- $\eta_{c}$ systems, and their results did not indicate any possibility for bound states or resonances in these channels. More lattice calculations of the excited baryon spectrum will be highly appreciated by the scientific community, anticipating the discovery of many more baryons in experiments like LHCb and Belle.

\section{Heavy exotics}

Doubly bottom and doubly charm tetraquarks: Early model calculations indicated that doubly heavy four quark systems in the heavy quark limit to be a promising platform to find stable tetraquark states [39]. This has received significant attention over the recent years both in phenomenological studies [40,41] and on the lattice [42-49]. A summary of various lattice and some non-lattice calculations can be found on the left side of Fig. 4. Lattice investigations remain exploratory in this regard, and except one, most lattice results are based on the ground-state mass estimates. Even though this is the case, qualitative inferences can be made for possible bound state scenarios. Only in Ref. [47], the authors perform an amplitude analysis to determine the actual binding energy, whereas in Refs. [42, 43] the authors determine static quark potentials in the presence of two light quarks on the lattice and search for bound state poles. In the doubly bottom axial-vector channel, all lattice investigations predict a deeply bound state with binding energy $O(100 \mathrm{MeV})$ for both $I=1(\bar{b} \bar{b} d u)$ and $I=1 / 2(\bar{b} \bar{b} s u)$. For $\bar{b} \bar{b} c u$, the lowest finite-volume level was found to be consistent with the threshold [45]. Similar investigations in the doubly charm sector have suggested the lowest finite-volume level immediately below or consistent with the 
elastic threshold. The $c c \bar{d} \bar{u}$ channel is related to the $T_{c c}$ reported by LHCb recently. A chiral and continuum extrapolated estimate for $\bar{c} \bar{c} d u$ ground-state in the finite-volume by ILGTI collaboration suggests possible binding energy of up to $23 \mathrm{MeV}$ [45]. However, a rigorous amplitude analysis of the finite-volume spectrum is highly desired to assess such near-threshold poles quantitatively.

Charmed-bottom tetraquarks: It is also interesting to investigate possible scenarios if one of the heavy quarks is replaced with a lighter heavy quark. Investigations in these systems have already been reported in Refs. [46, 48], which in conclusion claimed to observe no finite-volume levels below the elastic thresholds in any channels other than in the doubly bottom four quark systems. These investigations were performed on PACS-CS lattices with single lattice spacing $(\sim 0.09 \mathrm{fm})$. A recent lattice investigation by ILGTI collaboration utilizing up to three lattice spacings $(\sim 0.12$ $\mathrm{fm}, \sim 0.09 \mathrm{fm}$, and $\sim 0.06 \mathrm{fm}$ ) suggests that, on the fine lattice spacing, they find finite-volume levels that are quantitatively below the elastic threshold. They also observe that the interpolator basis commonly used for all these calculations is based on constraints in the heavy quark limit, whereas away from this limit, other allowed operators could be essential to determine the complete excitation spectrum reliably. In the center of Fig. 4, we present the light quark mass dependence of the finite-volume spectrum in the $\bar{b} \bar{c} d u$ axial-vector channel [in the range of $250 \mathrm{MeV}-800 \mathrm{MeV}$ pion mass] on the finest lattice. At all quark mass values, there is at least one level below the elastic threshold, unambiguously pointing to interesting physics near the threshold. The zero in the y-axis refers to the elastic threshold.

Heavy dibaryons : Several lattice groups have investigated dibaryon spectra. However, all were focussed on the light/strange dibaryons c.f. Refs. [50-52]. In a recent investigation [38], the authors have extracted the finite-volume spectrum of Deuteron-like heavy dibaryon systems looking for evidence of possible bound states in such system. Given that the Deuteron is a bound state in the physical world, bound states are naturally expected in its heavy analogues. The spectrum for a variety of dibaryon channels with flavor patterns $\Sigma_{c} \Xi_{c c}, \Omega_{c} \Omega_{c c}, \Sigma_{b} \Xi_{b b}, \Omega_{b} \Omega_{b b}$, and $\Omega_{c c b} \Omega_{c b b}$ in the axial-vector channel were reported in the article. They find strong indications for bound states in the dibaryon channels with $\Omega_{h}$ baryons, while robust inferences could not be made due to large systematic uncertainties in the other two channels. A summary plot of their findings is shown on the right side of Fig 4.

Except for a few calculations, all other lattice results presented above ignore the effects of any nearby strong decay thresholds. This is justified for most of the baryons discussed in the previous section. However, for the four-quark systems discussed in this section and the $\Sigma_{c}, \Sigma_{c}^{*}$ and $\Xi_{c}^{*}$ baryons that can decay via strong interactions, such a procedure is questionable. In these cases, a rigorous determination of the excitation spectra followed by a proper finite-volume analysis, such as discussed in the next section, is desired.

\section{Excited and exotic charmonium}

A large fraction of experimentally known heavy quark exotics is found in the charmonium spectrum. These are referred to as XYZs and have been discovered with properties contradicting the theoretical expectations from simple minded potential models. The first one in this family is $X$ (3872) discovered by Belle in 2003 [53]. Today, there are several such candidates with unexplained 
nature in the charm and the bottom sectors. A summary of efforts to find a theoretical description of these states can be found in Refs. [4, 54].

Many precision lattice calculations have been made for the ground-state charmonium masses, c.f. Ref. [21] and the references therein. Several groups have also determined the excited charmonium spectrum in the finite-volume [55-58]. However, rigorous investigations of the relevant strong interaction thresholds and scattering amplitudes are limited to a few. Two lattice calculations extracted a bound state pole associated with $X(3872)$ assuming $D \bar{D}$ elastic scattering [59, 60]. These works assumed the effects of couplings with other open scattering channels to be negligible. A similar investigation in the hidden charm $I=1$ sector indicated no signatures for the existence of any interaction in the low energy regime [61]. Recently HadSpec Collaboration has performed a detailed calculation to extract the finite-volume spectra in the rest frame for $I=1$ hidden charm [62]. They also arrived at a similar inference from their finite-volume spectra as in Ref. [61]. All of these lattice calculations were limited to the rest frame. In another letter by the HALQCD Collaboration, using their finite-volume formalism, they investigate the interactions between $\pi J / \psi$, $\rho \eta_{c}$ and $\bar{D} D^{*}$ and argues the charged $Z_{c}$ (3900) to be a threshold cusp [63].

In the vector charmonium spectrum, there are two bound states $J / \psi, \psi(2 S)$ and the $\psi(3770)$ resonance below $4 \mathrm{GeV}$. Given the sparse spectrum and the elastic nature of the $\psi(3770)$ resonance, this channel can be studied as the proof of principle. In the scalar charmonium, the situation is much less clear with a single bound state $\chi_{c 0}(1 P)$ and three exotic candidate resonances around the energy region $3.9 \mathrm{GeV}[64,65]$. Only one lattice investigation was performed, till 2019, for these channels assuming an elastic scattering of $D \bar{D}$ in the rest frame [66]. In what follows, we will discuss a recent calculation of these channels performed by the RQCD Collaboration in an extended lattice QCD setup. This new calculation utilizes two lattice QCD ensembles and works in three different inertial frames to extract the physics. The ensembles used have $m_{\pi} \sim 280 \mathrm{MeV}$ and $m_{K} \sim 467$ $\mathrm{MeV}$, and with spatial extents $L \sim 2 \mathrm{fm}$ and $L \sim 2.7 \mathrm{fm}$. The calculation was performed at two different values of charm quark masses to investigate the influence of the open-charm threshold.

In Figure 5, we present the results obtained for the vector charmonium spectrum in $D \bar{D}$ scattering in $l=1$ partial wave. Each finite-volume energy level can be associated with an infinitevolume phase shift for elastic scattering of spinless particles in a single partial wave. On the left, the $D \bar{D}$ scattering phase-shifts, $\left(a^{2} p^{3} \cot \left(\delta_{1}\right) / \sqrt{s}\right)$, are plotted as a function of the scattering momentum. The data points represent finite-volume spectra along the $\mathrm{x}$-axis and the $D \bar{D}$ scattering phase-shifts along the y-axis. The extracted $D \bar{D}$ scattering phase-shift is then fit with double pole parametrization of the scattering matrix, represented by the solid red curve and the associated errors by the blue band. The pole positions and residues of the singularities in the parametrized scattering matrix in the complex s-plane are extracted by analytic continuation. On the right, we present the extracted infinite-volume spectra along with the respective experimental numbers. It is evident from the plots that the extracted energies of the excitations remain mostly unaffected with unphysical threshold positions and are consistent with the experimental values.

In Figure 6, we present the results from the lattice investigation of scalar charmonium spectra in $D \bar{D}$ and $D_{s} \bar{D}_{s}$ multi-channel scattering. This investigation considers the energy region with a lower bound slightly below the $D \bar{D}$ threshold and an upper bound around $4.1 \mathrm{GeV}$. In the energy region around the open charm elastic threshold $(D \bar{D})$, a very shallow bound state pole is required to describe the observed finite-volume spectrum. The presence of such a pole is expected to cause a 

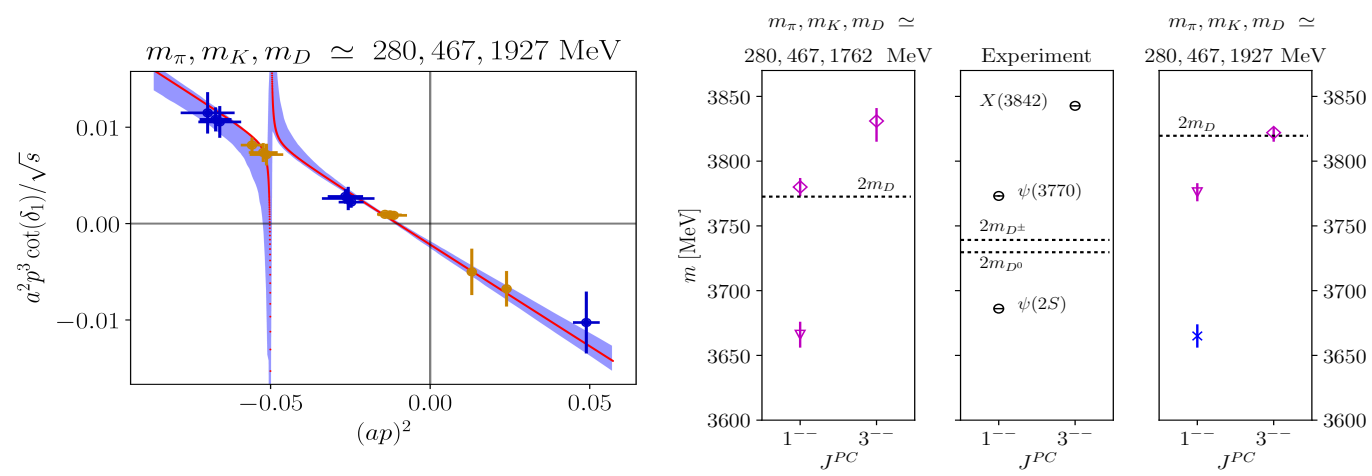

Figure 5: (Figures adapted from Ref. [67]) Left: $D \bar{D}$ scattering amplitude in the vector channels as a function of the square of scattering momentum. Results are shown for the heavier than physical charm quark mass. Right: The infinite-volume spectrum extracted from the finite-volume spectra are compared with the respective experimental values.
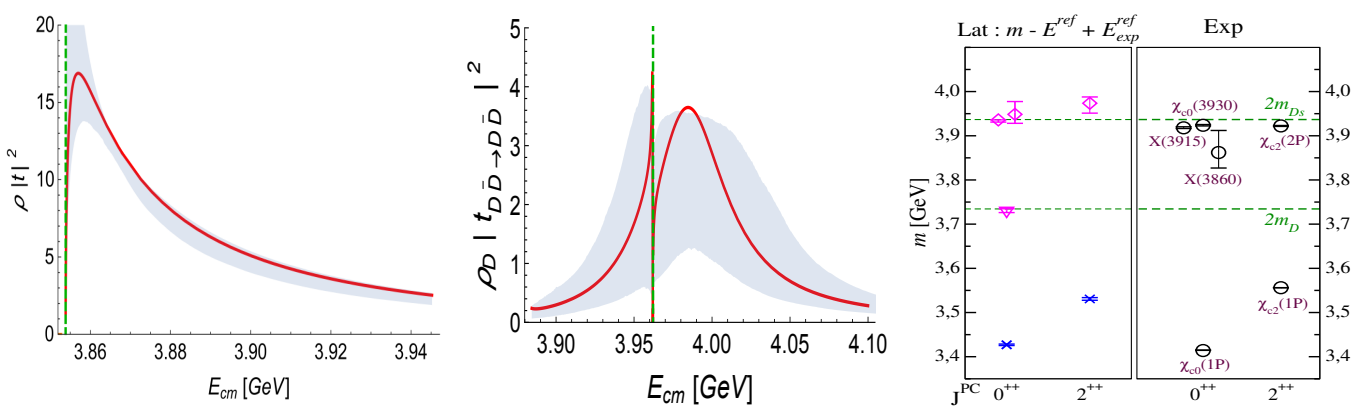

Figure 6: (Figures adapted from Ref. [68]) Left and center: $D \bar{D}$ scattering amplitude in the scalar channel as a function of the center of momentum energy, $E_{c m}$. On the left, the amplitude close to the $D \bar{D}$ threshold (vertical green dashed line) is shown. In the center, the amplitude in the energy region around $D_{s} \bar{D}_{s}$ threshold (vertical green dashed line) is plotted. Right: The extracted charmonium spectrum is compared with the experimental values.

rapid rise in the $D \bar{D}$ scattering amplitude immediately above the threshold, as shown in the left plot in Figure 6. Such a shallow bound state in $D \bar{D}$ channel was also proposed by phenomenological models such as in Refs. [69-71].

In the higher energy region starting from slightly below the $D_{s} \bar{D}_{s}$ threshold, the authors argue the presence of two pole singularities in the extracted scattering amplitude across the complex s-plane, that can have signatures on the real axis. One pole exists above the $D_{s} \bar{D}_{s}$ threshold in the Riemann sheet III (-,-) with large coupling to the $D \bar{D}$ channel. Such a pole is expected to reflect in the $D \bar{D}$ scattering amplitude as a vanilla resonance peak (see the center plot in Figure 6). Considering the extracted mass and its strong coupling to the $D \bar{D}$ scattering channel, the authors relate this state to the $\chi_{c 0}(3860)$ discovered recently by Belle [72].

Another pole is observed very close below the $D_{s} \bar{D}_{s}$ threshold in the Riemann sheet II with large coupling to $D_{s} \bar{D}_{s}$ channel. As a result of the interference with the pole above the $D_{s} \bar{D}_{s}$ threshold, this pole features in the $D \bar{D}$ amplitude as a prominent dip falling to zero below the $D_{s} \bar{D}_{s}$ threshold. This is also visible in the center plot in Figure 6. This dip in the $D \bar{D}$ crosssection is very similar to the dip in the $\pi \pi$ crosssection slightly below the $K \bar{K}$ threshold related to the $f_{0}(980)$ 
resonance [73]. Except at the $D_{s} \bar{D}_{s}$ threshold, where a kink appears, the $D \bar{D}$ amplitude remains smooth and continuous. The presence of this pole also causes a rapid rise in the $D \bar{D} \rightarrow D_{s} \bar{D}_{s}$ and $D_{s} \bar{D}_{s} \rightarrow D_{s} \bar{D}_{s}$ amplitudes immediately above the $D_{s} \bar{D}_{s}$ threshold. Resonance parameters of this pole share similar features to that of $\chi_{c 0}(3930) / \mathrm{X}(3915)$, both of which are below the $D_{s} \bar{D}_{s}$ threshold in the physical world. Most importantly, these states have narrow widths and were not observed in the $D \bar{D}$ final states, resulting from their large interaction with the $D_{s} \bar{D}_{s}$ channel.

In the right side of Figure 6, we present the extracted charmonium spectrum in the scalar channel together with the experimental observations. Several simplifying assumptions were made in this study of the excited charmonium spectrum, including heavier than physical light and charm quarks, lighter than physical strange quarks, and neglecting scattering channels $\left(J / \psi \omega\right.$ and $\left.\eta_{c} \eta\right)$. Furthermore, the scattering amplitudes were extracted with simple parametrizations, and the model dependence of the results was not investigated in detail. It would be exciting to see how these findings evolve when various approximations are relaxed in future lattice simulations.

\section{Summary}

Precision measurements of ground-state hadrons are now well established using lattice QCD methodology. We summarized various lattice results for ground-state charmed and bottom baryons. Recent highlights has been the $\Xi_{c c}$ baryon and the excited $\Omega_{c}$ baryons discussed in Section 2. Lattice predictions for the mass of $\Xi_{c c}$ baryon (and several other baryons) agree very well with the experimental value. We also discuss some of the recent lattice efforts in the heavy tetraquark and dibaryon sectors in Section 3. Various lattice studies points to the existence of strong interaction stable doubly bottom tetraquark system. More calculations are anticipated with regard to the doubly charm and charmed-bottom four quark systems.

In Section 4, we discuss the status of lattice investigations of charmonium excited states with special focus on the vector and scalar channels. While the studies of the vector channel serves as a proof of principle, the studies of scalar charmonium spectra are aimed at possible explanations of the low-lying scalar charmonium-like exotic candidates within the framework of QCD. The highlight in these studies is the observation of a shallow $D \bar{D}$ bound state and two other pole singularities possibly related to $\mathrm{X}(3860)$ and $\chi_{c 0}(3930) / \mathrm{X}(3915)$.

\section{Acknowledgments}

I thank Nilmani Mathur and Daniel Mohler for their careful reading of the manuscript.

\section{References}

[1] LHCв collaboration, R. Aaij et al., , Phys. Rev. Lett. 119 (2017) 112001 [1707. 01621].

[2] LHCв collaboration, R. Aaij et al., , Phys. Rev. Lett. 115 (2015) 072001 [1507.03414].

[3] LHCв collaboration, R. Aaij et al., , Phys. Rev. Lett. 118 (2017) 182001 [1703. 04639].

[4] A. Esposito, A. Pilloni and A. D. Polosa, Phys. Rept. 668 (2016) 1 [1611. 07920]. 
[5] S. L. Olsen, T. Skwarnicki and D. Zieminska, Rev. Mod. Phys. 90 (2018) 015003 [1708.04012].

[6] Proceedings, Lattice 2019: Wuhan, China, June 16-22 2019, SISSA, 2020.

[7] S. Aoki et al., Eur. Phys. J. C 80 (2020) 113 [1902.08191].

[8] S. Borsanyi et al., Science 347 (2015) 1452 [1406.4088].

[9] LHP collaboration, S. Basak et al., , Phys. Rev. D72 (2005) 074501 [hep-lat/0508018].

[10] J. J. Dudek et al., Phys. Rev. D82 (2010) 034508 [1004.4930].

[11] C. Michael, Nucl. Phys. B 259 (1985) 58.

[12] C. Liu, PoS LATTICE2016 (2017) 006 [1612 .00103].

[13] M. Padmanath, PoS LATTICE2018 (2018) 013 [1905 . 09651].

[14] M. Luscher, Commun. Math. Phys. 105 (1986) 153.

[15] HS collaboration, J. J. Dudek et al., , Phys. Rev. Lett. 113 (2014) 182001 [1406. 4158].

[16] R. A. Briceno, J. J. Dudek and R. D. Young, Rev. Mod. Phys. 90 (2018) 025001 [1706.06223].

[17] R. G. Edwards, PoS LATTICE2019 (2020) 253.

[18] M. T. Hansen and S. R. Sharpe, Ann. Rev. Nucl. Part. Sci. 69 (2019) 65 [1901.00483].

[19] M. Mai, M. Döring and A. Rusetsky, Eur. Phys. J. ST 230 (2021) 1623 [2103. 00577].

[20] B. Hoerz, PoS LATTICE2021 (2021) .

[21] C. DeTar et al., Phys. Rev. D 99 (2019) 034509 [1810.09983].

[22] LHCв collaboration, R. Aaij et al., , 1807.01919.

[23] SELEX collaboration, M. Mattson et al., , Phys. Rev. Lett. 89 (2002) 112001 [hep-ex/0208014].

[24] N. Mathur, M. Padmanath and S. Mondal, 1806.04151.

[25] M. Padmanath and N. Mathur, Phys. Rev. Lett. 119 (2017) 042001 [1704. 00259].

[26] E. B. Gregory et al., Phys. Rev. D 83 (2011) 014506 [1010 . 3848].

[27] R. J. Dowdall et al., Phys. Rev. D86 (2012) 094510 [1207 . 5149].

[28] Z. S. Brown, W. Detmold, S. Meinel and K. Orginos, Phys. Rev. D90 (2014) 094507 [1409.0497].

[29] M. Padmanath et al., Phys. Rev. D90 (2014) 074504 [1307.7022]. 
[30] M. Padmanath et al., Phys. Rev. D91 (2015) 094502 [1502 . 01845].

[31] M. Padmanath and N. Mathur, in Proceedings, 7th International Workshop on Charm Physics, CHARM 2015: Detroit, USA, May 18-22, 2015, 2015, 1508. 07168.

[32] S. Meinel, Phys. Rev. D85 (2012) 114510 [1202 . 1312].

[33] M. Padmanath et al., PoS LATTICE2014 (2015) 084 [1410 . 8791].

[34] M. Padmanath et al., in Proceedings, Charm 2013, 2013, 1311.4806.

[35] LHCв collaboration, R. Aaij et al., , 2107.03419.

[36] M. Alberti et al., Phys. Rev. D95 (2017) 074501 [1608.06537].

[37] U. Skerbis and S. Prelovsek, Phys. Rev. D 99 (2019) 094505 [1811. 02285].

[38] P. Junnarkar and N. Mathur, Phys. Rev. Lett. 123 (2019) 162003 [1906. 06054].

[39] J. Carlson, L. Heller and J. A. Tjon, Phys. Rev. D37 (1988) 744.

[40] M. Karliner and J. L. Rosner, Phys. Rev. Lett. 119 (2017) 202001 [1707 . 07666].

[41] E. J. Eichten and C. Quigg, Phys. Rev. Lett. 119 (2017) 202002 [1707. 09575].

[42] P. Bicudo, J. Scheunert and M. Wagner, Phys. Rev. D95 (2017) 034502 [1612 . 02758].

[43] P. Bicudo et al., Phys. Rev. D96 (2017) 054510 [1704 . 02383].

[44] A. Francis et al., Phys. Rev. Lett. 118 (2017) 142001 [1607.05214].

[45] P. Junnarkar, N. Mathur and M. Padmanath, Phys. Rev. D 99 (2019) 034507 [1810 . 12285].

[46] A. Francis, R. J. Hudspith, R. Lewis and K. Maltman, Phys. Rev. D 99 (2019) 054505 [1810.10550].

[47] L. Leskovec, S. Meinel, M. Pflaumer and M. Wagner, Phys. Rev. D 100 (2019) 014503 [1904.04197].

[48] R. J. Hudspith, B. Colquhoun, A. Francis, R. Lewis and K. Maltman, Phys. Rev. D 102 (2020) 114506 [2006. 14294].

[49] P. Mohanta and S. Basak, Phys. Rev. D 102 (2020) 094516 [2008. 11146].

[50] NPLQCD collaboration, S. R. Beane et al., , Phys. Rev. Lett. 106 (2011) 162001 [1012.3812].

[51] HAL QCD collaboration, T. Inoue, N. Ishii, S. Aoki, T. Doi, T. Hatsuda, Y. Ikeda et al., , Phys. Rev. Lett. 106 (2011) 162002 [1012 . 5928].

[52] J. R. Green, A. D. Hanlon, P. M. Junnarkar and H. Wittig, 2103. 01054. 
[53] Belle collaboration, S. K. Choi et al., , Phys. Rev. Lett. 91 (2003) 262001 [hep-ex/0309032].

[54] S. L. Olsen, in Proceedings, 53rd International Winter Meeting on Nuclear Physics (Bormio 2015): Bormio, Italy, January 26-30, 2015, 2015, 1511.01589.

[55] G. S. Bali, S. Collins and C. Ehmann, Phys. Rev. D84 (2011) 094506 [1110 . 2381].

[56] HS collaboration, L. Liu et al., , JHEP 07 (2012) 126 [1204 . 5425].

[57] D. Mohler, S. Prelovsek and R. M. Woloshyn, Phys. Rev. D 87 (2013) 034501 [1208 . 4059].

[58] M. Padmanath et al., Phys. Rev. D 99 (2019) 014513 [1811.04116].

[59] S. Prelovsek and L. Leskovec, Phys. Rev. Lett. 111 (2013) 192001 [1307. 5172].

[60] M. Padmanath, C. B. Lang and S. Prelovsek, Phys. Rev. D92 (2015) 034501 [1503. 03257].

[61] S. Prelovsek and L. Leskovec, Phys. Lett. B727 (2013) 172 [1308 . 2097].

[62] HS collaboration, G. K. C. Cheung et al., , JHEP 11 (2017) 033 [1709. 01417].

[63] HAL QCD collaboration, Y. Ikeda et al., , Phys. Rev. Lett. 117 (2016) 242001 [1602 . 03465].

[64] Belle collaboration, K. Chilikin et al., , Phys. Rev. D95 (2017) 112003 [1704 . 01872].

[65] LHCв collaboration, R. Aaij et al., , JHEP 07 (2019) 035 [1903. 12240].

[66] C. B. Lang et al., JHEP 09 (2015) 089 [1503.05363].

[67] S. Piemonte, S. Collins, D. Mohler, M. Padmanath and S. Prelovsek, Phys. Rev. D 100 (2019) 074505 [1905 . 03506].

[68] S. Prelovsek et al., JHEP 06 (2021) 035 [2011. 02542].

[69] D. Gamermann, E. Oset, D. Strottman and M. J. Vicente Vacas, Phys. Rev. D 76 (2007) 074016 [hep-ph/0612179].

[70] C. Hidalgo-Duque, J. Nieves, A. Ozpineci and V. Zamiralov, Phys. Lett. B 727 (2013) 432 [1305.4487].

[71] V. Baru, E. Epelbaum, A. A. Filin, C. Hanhart, U.-G. Meißner and A. V. Nefediev, Phys. Lett. B 763 (2016) 20 [1605.09649].

[72] Belle collaboration, K. Chilikin et al., , Phys. Rev. D 95 (2017) 112003 [1704.01872].

[73] R. A. Briceno, J. J. Dudek, R. G. Edwards and D. J. Wilson, Phys. Rev. D 97 (2018) 054513 [1708.06667]. 